\title{
Aprendiendo a regular la acción conjunta: el formato de dar-y-tomar *
}

\author{
IGNASI VILA \\ JAVIER ZANÓN \\ Universidad de de Barcelona
}

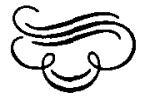

\section{Resumen}

El desarrollo de mecanismos para regular la acción conjunta es uno de los objetivos comunicativos más tempranos que el niño debe conseguir a lo largo de sus dos primeros años de vida. El contexto de dar-y-tomar aparece como una de las situaciones naturales en las que el niño conoce y aprende el dominio del curso de la acción. La interacción en dicha situación cumple una doble función, semántica y prágmatica, a la vez que permite la adquisición de las primeras distinciones gramaticales. En este sentido, el estudio del dar-y-tomar muestra el comienzo y desarrollo de las técnicas de requerimiento, la elaboración lingüistica y conceptual de los términos asociados a sus segmentos de acción y el uso de los primeros pares deicticos. El artículo estudia el inicio y el desarrollo de este formato en dos niños y una niña durante sus dos primeros años de vida en situaciones interactivas con sus madres.

\section{Abstract}

The development of mechanisms for the regulation of joint action constitutes one of the earliest comunicative goals which infants have to achieve during their first two years of life. The "give-and-take" format, outstands as one the "natural contexts" where the baby knows and learns for the first time the control of the course of action. Interaction within this situation serves a double function, semantic and pragmatic, as well as being a structural element for grammar acquisition. In this respect, the study of the "give-and-takes format shows the beginning and development of query techniques, together with the linguistic and conceptual elaboration of the terms associated to first actions and the use of the first deictic pairs. Our article studies the beginning and development of this format in three babies and its interaction with adults during their first two years of life.

* Esta investigación ha sido subvencionada por la Comisión Asesora de Investigación Científica y Técnica del Ministerio de Educación.

Una primera versión de este artículo fue leída como comunicación en la reunión anual de la Sección de Psicología del Desarrollo de la British Society of Psychology celebrada en la University of Exeter en septiembre de 1986.

Dirección de los autores: Universidad de Barcelona. Facultad de Psicología. Departamento de Psicología Evolutiva y de la Educación. Ava. de Chile, zona universitaria de Pedralbes, 08028 Barcelona. 
El estudio de los prerrequisitos que anteceden a la aparición del lenguaje evidencia la importancia que tiene el dominio del curso de la acción por parte del aprendiz en relación a la construcción de su primer lenguaje. Bruner $(1975,1977)$ hipotetizó que el curso de la acción y la estructura lingüistica eran simétricos de forma que en ambos dominios aparecían nociones que se correspondían unas a las otras. Así, el dominio del curso de la acción comporta nociones como agente, receptor, acción, locativo, etc., y a la estructura lingüística, siguiendo la gramática del caso, subyacen nociones semejantes. Además, Bruner, apoyándose en el orden de las palabras, concluía que a todo lenguaje subyacía una estructura universal del tipo agente-acción-objeto (Juan come un bistec) que era reconocida por el niño en la medida en que dominaba dichas nociones en el curso de la acción que respondía a dicha estructura. Ciertamente, Bruner no tenían en cuenta que si bien en lenguas como el castellano el orden de las palabras determina el significado y éste se corresponde con el curso de la acción, existen otras lenguas que o bien el orden de las palabras es variable, explicitándose el significado mediante flexiones sobre las palabras, o bien funcionan ambos mecanismos como en el caso del euskera. Sin embargo, independientemente de las incorrecciones incluidas en su propuesta, otros autores (Bickerton, 1981; Slobin, 1986) han reformulado dicha hipótesis.

Bickerton, tras sus trabajos sobre la creolización, propone que la especie humana está programada biológicamente para establecer una serie de distinciones en la realidad, así como para escoger las distinciones lingüísticas que se corresponden con ellas. En concreto, propone que los humanos reconocemos las distinciones entre: a)sucesos específicos y no específicos; b) estados y procesos; $c$ ) hechos puntuales y continuos, $y \mathrm{~d}$ ) acciones causales y no causales. Justamente, el adulto y el niño se concentran en la acción conjunta tanto en estas distinciones como en las formas lingüísticas que las marcan. Por su parte, Slobin (1986) plantea la misma hipótesis en otros términos. Considera que la adquisición del lenguaje es en definitiva un proceso a lo largo del cual el aprendiz construye relaciones entre formas lingüísticas y los significados que expresan. Por tanto, su explicación de la adquisición del lenguaje reside en hipotetizar los distintos procedimientos al alcance del niño que le permiten establecer relaciones forma-significado. En este sentido, propone la existencia de escenas prototípicas que define como aquellas que primero reciben una marca gramatical en la gramática Básica del Niño, siendo esta última universal al menos en cuanto a los principios con que opera. El término escena, tal y como es utilizado por Slobin, se relaciona directamente con el mismo significado con que Fillmore utiliza dicho término. En consecuencia, Slobin reconoce que las escenas ocurren regularmente como parte de actividades y percepciones frecuentes y claramente llamativas. De hecho, como señala el mismo Slobin, este concepto ha recibido distintos términos en la psicologia del desarrollo. Savasir y Gee (1982) emplean el término «tipos de actividad» y Claudia de Lemos (1981), parodiando a Bruner, les da el nombre de «formatos interactivos». Pero, sin dudas, fue Bruner $(1982,1983 a)$ quien con el nombre de «formato" destacó el carácter reglado y rutinario de estas actividades que pueden clasificarse como de atención y de acción conjunta. Slobin cree que en las escenas aparecen un estrecho conjunto de nociones básicas que el aprendiz del lenguaje construye a la vez que reconoce sus marcas lingüísticas al em- 
plear los adultos en dichas situaciones repetidamente las formas lingüísticas que denotan dichas nociones salientes. Slobin ejemplifica su propuesta con la "escena de actividad manipulativa", que consiste en situaciones de acción conjunta entre el adulto y el niño como el dar-y-tomar, el construir$y$-tirar o semejantes. Las actividades manipulativas que se desarrollan en dichos formatos, en el sentido de Bruner, comportan una serie de nociones relacionadas, como son el concepto de agente, los conceptos de cambio de estado o de lugar o la noción de causalidad. Todas ellas forman en conjunto una representación conceptual prototípica semejante a la noción de gestalt que, en boca de otros autores (Nelson, 1983; Vila, 1984 y 1987), ha recibido el nombre de script. Así, las primeras formas lingüísticas conocidad como functors se relacionan directamente con este tipo de escenas o con alguno de sus elementos claramente perceptibles. De esta forma, en la medida en que Slobin considera que este tipo de escenas representan tipos de actividad prototípica, los niños y las niñas gramaticalizarán las mismas nociones independientemente de la lengua que estén aprendiendo.

Así, la acción conjunta, acción en la que se implican un adulto y un aprendiz del lenguaje en un marco interactivo, aparece como uno de los dominios en los que el niño puede organizar el campo semántico y sus marcadores gramaticales. Pero la acción conjunta no sólo posibilita dicha organización, sino que además constituye una de las áreas más relevantes para que el niño domine determinadas técnicas en relación al uso del lenguaje. En ella se destacan las técnicas para requerir o para ofrecer o se establecen los límites para el uso de determinados pares deícticos que modifican su referencia en función de la perspectiva del hablante en el discurso. Es por eso por lo que el dominio de la acción conjunta representa uno de los ámbitos que el infante debe conquistar para acceder y progresar en el uso del lenguaje.

Nuestro trabajo pretende ejemplificar dichas propuestas en uno de los ámbitos de la acción conjunta: la situación de dar-y-tomar. De hecho, más allá de las hipótesis explicitadas, prácticamente no existen trabajos empíricos que muestren el funcionamiento de la acción conjunta y los avances por parte del niño en el dominio de las técnicas que la regulan.

\section{METODO}

\section{Sujetos}

Nuestros sujetos, M.' del Mar, Roger y Emilio, pertenecen a familias de nivel sociocultural medio/alto. M. ${ }^{2}$ del Mar fue registrada a partir de los 14 meses, Emilio a partir de los 10 meses y Roger desde los 5 meses. Los tres fueron seguidos hasta los dos años. Emilio emplea exclusivamente el castellano en sus comunicaciones, mientras que $\mathrm{M}^{2}$ del Mar y Roger emplean el catalán con sus padres y el castellano con sus madres.

\section{Procedimiento}

Los registros se realizaron cada dos/tres semanas, grabando en magnetoscopio 30/45 minutos de interacción adulto-niño en sus propias casas. La mayor parte de sesiones recayeron en las madres, aunque en ocasiones tam- 
bién participaron los padres u otros adultos. Los adultos no recibieron ninguna consigna sobre el modo de interactuar y únicamente se les dijo que realizábamos un estudio sobre el bilingüismo familiar. Todo el material fue transcrito según un sistema de cuatro columnas en el que se especificaba el lenguaje del adulto, el lenguaje del niño, las conductas no verbales del adulto y las conductas no verbales del niño (Siguan, Colomina, Vila, 1986).

Consideramos situaciones de dar-y-tomar todas aquellas interacciones en las que el adulto y el niño actuaban conjuntamente sobre un objeto en una actividad de intercambio. Este contexto se inicia tempranamente de forma que a los 12 meses está prácticamente constituido. Por eso, los resultados tienen en cuenta especialmente los datos de Roger, añadiéndose los de M.' del Mar y Emilio para ilustrar determinadas afirmaciones.

\section{RESULTADOS}

Los resultados ocupan varios apartados. En primer lugar, destacamos los aspectos globales del contexto referidos a su constitución, evolución e implicación de los participantes. En segundo lugar, explicitamos las implicaciones del contexto para la elaboración lingüística y conceptual de los términos asociados a los distintos segmentos implicados en este tipo de acción conjunta. Finalmente, estudiamos específicamente los problemas con que se encuentran nuestros sujetos para emplear correctamente algunos términos lingüísticos como son los pares deícticos.

El inicio de las situaciones de dar-y-tomar en el dúo formado por Roger y su madre aparecen a la edad de 0;5,5 cuando el adulto muestra un objeto de la vida habitual del niño, recreándose en él, de forma que Roger le preste atención.

\section{OBSERVACION I: ROGER $(0 ; 5,5)$}

(Roger está sentado en su silla después de comer)

(levanta la correa que ata $\mathrm{M}: \mathrm{mira} / \mathrm{R}$ a R. a la silla)

(muestra la correa a R.) M: toma/ la correa de tu silla que te gusta tanto/

\section{$\mathrm{R}$}

(agita la correa delante de $\mathrm{M}: \mathrm{mira} / \mathrm{x} / \mathrm{R}$

la cara de $\mathrm{R}$.)

$\begin{array}{lll}\text { (suelta la correa) } \quad \text { M:oh/ qué bonita es/ } & \begin{array}{l}\text { R: ah(:)/ } \\ \text { correa }\end{array} & \begin{array}{l}\text { (alarga el brazo hacia } \\ \text { la correa) } \\ \text { (coge la correa, }\end{array} \\ & \begin{array}{ll}\text { correa ) } \\ \text { R: balbuceo/ } \\ \text { (tiene la correa en la } \\ \text { correa }\end{array}\end{array}$

En estos primeros momentos del contexto la madre de Roger dirige su actividad con el objeto de adiestrar a su hijo en la recepción de objetos. Los procedimientos que emplea se centran básicamente en el mantenimien- 
to de la atención de su hijo sobre el objeto que éste debe alcanzar. Así, emplea conductas como agitar y acercar el objeto al niño, tocar con el objeto el cuerpo de Roger, además de explicitar lingüísticamente su ofrecimiento mediante términos como ten o toma. El lenguaje que emplea la madre de Roger en estos momentos exclusivamente cumple las funciones de ofrecer. Unicamente cuando se inicia la interacción emplea vocativos de atención que tienen como objeto controlar la atención de su hijo. Finalmente, una vez que Roger alcanza el objeto y lo mantiene entre sus manos, su madre acostumbra a realizar algún comentario para mantener la atención de su hijo sobre el objeto.

De estas primeras observaciones, el «intento de alcanzar» de Roger no es un requerimiento explícito, ya que en ningún otro momento busca con la mirada a su madre, sino que la centra exclusivamente sobre el objeto. Es decir, aún es incapaz de contemplar al adulto como un posible agente en la situación de intercambio.

Un mes después, la madre de Roger introduce un nuevo mecanismo para ejercitar a su hijo en la recepción de objetos.

\section{OBSERVACION II: Roger $(0 ; 6,1)$}

(Roger y su madre juegan con un perro de peluche)

(mueve el perro) $M:$ mira/ $R$
$\begin{aligned} & \text { (acerca el perro a R.) } \\ & \text { M: toma/ } R\end{aligned}$
(aleja el perro de la cara $M:$ venga que te
de R.)
(aleja y acerca el perro a $M:$ venga/ $R$
la cara de $R$. con un mo-
vimiento de vaivén)

(tiende el brazo hacia

el perro, perro )

(ofrece el perro)

$\mathrm{M}$ : venga/ toma/ $\mathrm{R}$

\author{
(quieto, perro ) \\ (quieto, perro )
}

(coge el perro, perro )

A partir del sexto mes de vida de Roger, su madre incluye en su actividad una gama de conductas que tienen como objeto incitar la acción de su hijo. Así, establece series de movimientos para mantener su atención tales como acercar y alejar el objeto, a la vez que incluye comportamientos lingüísticos (venga, va, etc.) que justamente se emplean para incitar a la acción. Por su parte, Roger emplea conductas semajantes a las observadas anteriormente en el tiempo. Continúa sin considerar al adulto como un agente y, por tanto, no aparece ningún intento de buscar su ayuda para completar el intento de alcanzar.

A los 0;6,13 aparece un nuevo cambio en la conducta de la madre de Roger. A lo largo de una situación interactiva, pautada en forma de juego, en la que ambos golpean sucesivamente un cojín, la madre de Roger «hace saber» a éste que puede ser agente de acciones. La estructura de la situación, que no es propiamente un dar-y-tomar, se marca lingüísticamente en 
el baby-talk del adulto. El interés de esta situación reside en que antecede a los intentos maternos para convertir a Roger en un donante de objetos, estructurándose de una forma muy semejante a como posteriormente se lo hará saber su madre en la situación de dar-y-tomar.

La madre de Roger emplea un vocativo de atención para llamar la atención de su hijo sobre una acción suya (golpear el cojín con la mano). Luego, da el turno a Roger mediante el término tú, a la vez que «andamia» su improbable conducta cogiéndole la mano y llevándola hacia el cojín. Seguidamente marca la acción de Roger con el término pam. Es decir, a la vez que dirige la mano de Roger hacia el cojín para que lo golpee, cada vez que ésta toca el cojín dice pam. Esto lo repite un buen número de veces, de forma que su repetición permanente posibilita que los términos lingüísticos se incorporen como partes del conjunto de la situación, ayudando a pautarla, a la vez que la marcan y la seleccionan de forma que el uso de uno ellos comporta la toma de un turno en la interacción y la correspondiente invitación al compañero para que tome el suyo.

El estudio de los términos empleados por la madre de Roger en este período reafirman las observaciones anteriores. Esta dedica todos sus esfuerzos en conseguir que Roger sea un buen receptor. La Tabla I presenta dicha distribución entre los 5 y los 6 meses y medio.

TABLA I

Distribución del lenguaje de la madre de Roger en el juego de dar-y-tomar entre los 0,5 y los $0 ; 6,13$

\begin{tabular}{lcccccc}
\hline Edad & Ten & Toma & Toma el/la X & Toma tu X & Toma-Y & TOTAL \\
\hline $0 ; 5,5$ & 0 & 3 & 1 & 0 & 0 & 4 \\
$0 ; 6,1$ & 1 & 42 & 1 & 2 & 1 & 47 \\
$0 ; 6,13$ & 1 & 11 & 1 & 0 & 1 & 14 \\
\hline TOTAL & 2 & 56 & 3 & 2 & 2 & 65 \\
\hline
\end{tabular}

* $X$ es un nominal.

$*$ Y es un pronombre.

Toma es el principal recurso lingüistico que emplea la madre de Roger para marcar sus ofrecimientos, utilizándolo generalmente en forma aislada para que pueda ser claramente percibido por Roger.

A los 0;8,0 aparecen innovaciones en el desarrollo del formato.

OBSERVACION III: Roger $(0 ; 8,0)$

(Roger y su madre juegan con un manojo de llaves)

(pone las llaves al alcance $M$ : sí/venga/fuerte/ $/ x /$ de R.)
$\mathrm{R}$

$M$ : venga/x/ R (extiende el brazo hacia las llaves, llaves,

(ríe, extiende el brazo hacia las llaves, llaves ) 
M: fuerte que me las lle$\mathrm{vo} / \mathrm{R}$

M: corre que te las quito $/ \mathrm{ahy} / \mathrm{x} / \mathrm{x} / \mathrm{x} / \mathrm{x} / \mathrm{x} / \mathrm{x} / \mathrm{R}$

(baja el brazo con las lla- M: huy/ $\mathrm{R}$ ves)

(tiende la mano abierta $\mathrm{M}$ : dame/dame/ $\mathrm{R}$ hacia R.) (ríe, estira de las llaves, llaves )

(rie, estira de las llaves, llaves )

(estira de las llaves, llaves ) (se queda con las llaves, llaves )

(toca la mano de $\mathrm{M}$. con las suyas, mano de M )

(coge una llave, estira ha- $M$ : venga $/ x / R$ cia arriba)

(ríe, estira de las llaves, llaves )

Por un lado, los esfuerzos maternos comienzan a dar resultado y Roger comienza a «conocer» al adulto como un medio para conseguir alcanzar un objeto, es decir, como agente de una acción, a la vez que se conoce como el receptor de la misma. Roger ya no se limita a extender el brazo para alcanzar un objeto que su madre le presenta, sino que comienza a utilizar ese procedimiento de forma independiente y extiende el brazo hacia el objeto de su interés, lo haya o no presentado su madre. Esta conducta se acompaña además de una mirada alternante entre el objeto y el adulto, buscando Roger el soporte explícito de su madre en la consecución de su objetivo. Es el inicio de las técnicas de requerimiento.

Por otro, la madre de Roger potencia al máximo las posibilidades de Roger como agente de una acción. Así, diversifica sus incitaciones a la acción, que ya no se limitan al término toma (en este período emplea producciones como cógelo, cógelo fuerte, tira fuerte, corre, etc.), marca lingüísticamente sus requerimientos mediante el término dame y disminuye ostensiblemente la fase de mantenimiento de atención empleada en etapas anteriores para conseguir que su hijo atendiera al tipo de tarea propuesta.

En este momento la relación entre ofrecimientos y requerimientos maternos comienza a igualarse, de forma que el formato comienza a adquir todas sus características. La Tabla II presenta dicha relación desde los 5 a los 11 meses.

TABLA II

Distribución de los sujetos

\begin{tabular}{|c|c|c|c|}
\hline Edad & Ofrecimiento & Requerimiento & TOTAL \\
\hline $\begin{array}{l}0 ; 5,5 \\
0 ; 6,1 \\
0 ; 6,13 \\
0 ; 8,0 \\
0 ; 10,22\end{array}$ & $\begin{array}{r}4 \\
48 \\
14 \\
24 \\
15\end{array}$ & $\begin{array}{r}0 \\
0 \\
0 \\
0 \\
3 \\
11\end{array}$ & $\begin{array}{r}4 \\
48 \\
14 \\
27 \\
26\end{array}$ \\
\hline TOTAL & 105 & 14 & 119 \\
\hline
\end{tabular}


La actividad materna provoca cambios importantes en el conjunto de la interacción. Roger, a partir de estos momentos, es capaz de iniciar ciclos de intercambio y, por tanto, como ya hemos señalado, la situación de dary-tomar comienza a adoptar una estructura simétrica. Por ejemplo, la siguiente observación muestra el inicio del auténtico dar-y-tomar.

OBSERVACION IV: Roger $(0 ; 10,22)$

(Roger y su madre juegan con una pelota)

(mueve la cabeza hacia M: tíramela/ $\mathrm{R}$ adelante)

(coge la pelota, $\bar{M}$ )

(coge la pelota)

$M$ : venga/ pelota

(empuja la pelota, pelota )

(empuja la pelota hacia $M:$ pumba/ pelota R.)

$\begin{array}{ll} & \text { (coge la pelota, } \overline{\mathrm{M}} \text { ) } \\ & \text { (empuja la pelota, } \\ & \text { (quieto, pelota) } \\ & \text { (quieta }) \\ \text { (quieto, } & \\ \text { pelota) } & \end{array}$

(se estira hacia adelante)

$\mathrm{M}:$ pumba/ pelota

$\begin{array}{r}\text { R: eaual } \\ \text { Pelota } \\ \hline\end{array}$

(empuja la pelota,

(coge la pelota)

(quieto, pelota)

(empuja la pelota hacia M: pumbal pelota

R.)

El interés de Roger ya no reside en retener el objeto alcanzado, sino en participar en las acciones en que es traspasado rápidamente. El tiempo que el niño mantiene el objeto entre sus manos se reduce drásticamente y comienzan a aparecer producciones lingüísticas adyacentes a las fases de coger $\mathrm{y}$ ofrecer. El adulto, por su parte, reduce aún más la fase de ofrecimiento al eliminar casi definitivamente los procedimientos de mantenimiento de la atención y diversifica sus procedimientos lingüísticos que se relacionan con la fase de requerimientos tras incorporar términos como, por ejemplo, tira, además de continuar empleando dame.

A los $0 ; 11,9$, Roger emplea sus primeras vocalizaciones en el juego de dar-y-tomar.

\section{OBSERVACION V: Roger $(0 ; 11,9)$}

(Roger y su madre interactúan con diversos juguetes)

(mueve la mano extendi- $\mathrm{M}$ : dame $\mathrm{R}$ da hacia R.)

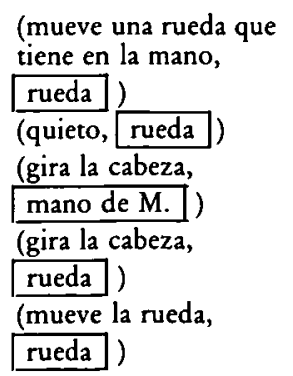


(extiende la mano hacia M: Roger/ dame/ $\mathrm{R}$ R.)

(levanta la rueda, rueda )

R: tál

rueda

(da la rueda a M.)

(coge la rueda)

M: gracias/ $\mathrm{R}$

(acerca su cara a R.)

M: gracias/ $\mathrm{R}$

(ofrece la rueda a $R$.)

M: toma/ para ti/ $\mathrm{R}$

M: ya está/ toma/ para ti/

$\mathrm{R}$

M: vale/ para ti/ $\mathbf{R}$
$\mathrm{R}: \mathbf{t a ́} / \cdot$

$\mathrm{R}:$ ya ta/ $\mathrm{M}$ (extiende el brazo)

\author{
(quieto, $\mathrm{M}$ ) \\ (quieto, $\mathrm{M}$ )
}

(quieto, $\mathrm{M}$ )

(gira la cabeza, $\square$ )

(gira la cabeza, M)

(toca la rueda, rueda)

Roger emplea tá como reducción de ya está en el momento de traspasar el objeto a su madre y como rechazo a continuar la interacción. Posteriormente, vuelve a emplear dicha fórmula, aunque más clara fonéticamente, cuando alcanza el objeto que le ofrece su madre. El uso del término en el formato de dar-y-tomar ejemplifica la aparición de las primeras producciones lingüísticas. De hecho, la madre de Roger emplea la fórmula ya está en numerosas situaciones marcando siempre el final de una acción. Así, emplea la fórmula tanto en situaciones ligadas a necesidades biológicas de Roger (comida, lavado, vestido, etc.) como en situaciones de juego. Roger, en estos momentos, emplea dicha fórmula en situaciones como, por ejemplo, cuando se acaba la comida en el plato o semejantes, de forma que tiende a generalizar su uso a otras situaciones en las que se marca el final de una acción o de un estado. Por eso, no es extraño que, reconociendo ya los diferentes segmentos del dar-y-tomar, emplee tá para marcar el final de cada uno de ellos. Así emplea ya está tanto cuando traspasa un objeto como cuando lo alcanza. Pero, además, también emplea este procedimiento para establecer que no quiere continuar jugando. Es decir, para regular justamente el final de la interacción. En este caso, su madre reconoce su intención, pero continúa animándole a participar en el juego, ofreciéndole nuevamente el objeto.

La conducta lingüística de la madre de Roger también se modifica ostensiblemente. En estos momentos introduce una amplia gama de formas pronominales que tienen una referencia deíctica y que especifican tanto sus ofrecimientos como sus requerimientos, así como sus incitaciones a la acción (para ti). adulto.

Un mes más tarde, Roger se acopla a requerimientos más complejos del 
OBSERVACION VI: Roger $(1 ; 0,8)$

(Roger tiene un mechero en la mano)

(tiende la mano abierta M: a ver/Roger/a ver/ $\mathrm{x} /$ hacia R.)

(tiende la mano abierta $\mathrm{M}$ : déjamelo/ $\mathrm{x} / \mathrm{R}$ hacia R.)

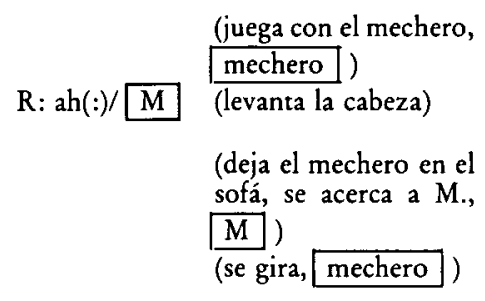

(P. da el mechero a R.) P: dona-li a la mama («dáselo a mamám/ corre/ $\mathrm{R}$

(coge el mechero, mechero )

(se gira hacia M. con el mechero en la mano, M )

$\mathrm{R}: \mathrm{ah} / \mathrm{M}$ (da el mechero a $\mathrm{M}$.)

(coge el mechero) $\mathrm{M}:$ gracias/ $\mathrm{R}$

Una vez que Roger domina las reglas del dar-y-tomar puede implicarse en situaciones en las que participan más de dos sujetos, lo cual diversifica la cantidad y la calidad de la acción conjunta, permitiendo una mayor amplitud pronominal, así como la incorparación de términos en lugar más diversos.

Una vez que Roger se adecua sin problemas a las reglas del dar-y-tomar, su madre introduce nuevas formas de requerimiento.

OBSERVACION VII: Roger $(1 ; 1,21)$

(Roger y su madre juegan con un pollito)

(muestra el pollito a M., M )

(extiende la mano abierta $\mathrm{M}$ : ¿̨me lo das?/ $\mathrm{R}$

hacia R.)

(tiende el pollito a M., M )

Las marcas lingüísticas comienzan a ser diferentes. El adulto introduce los mecanismos lingüísticos explícitos de requerimiento como son las preguntas, posibilitando su reconocimiento por parte de Roger al acompañarlos de gestos explícitos para requerir. El uso de estos procedimientos, tal y como muestra la Tabla III, aumenta de forma importante en las madres de nuestros tres sujetos a partir de la edad de $\mathbf{1 5}$ meses de sus hijos. 
TABLA III

Incremento de los requerimientos emitidos en tono interrogativo por parte de las madres de M.` del Mar, Roger y Emilio entre los 12 y los 18 meses en el juego de dar-y-tomar

\begin{tabular}{ccc}
\hline $\begin{array}{c}\text { Requerimientos } \\
\text { totales }\end{array}$ & $\begin{array}{c}\text { Requerimiento en } \\
\text { tono interrogativo }\end{array}$ & $\%$ \\
\hline
\end{tabular}

12-15 meses

Madre de M.2 del Mar

Madre de Roger

Madre de Emilio

7
38

14
0

2,63

7,14

\section{$15-18$ meses}

Madre de M. del Mar

A medida que avanza el formato en el habla del adulto aparecen nuevos términos para regular la acción conjunta. Muñoz y Vila $(1985,1986)$ y González y Vila (1986) muestran la importancia del contexto en la aparición de los pronombres personales y posesivos en el habla de M. ${ }^{2}$ del Mar y Emilio. Los autores afirman que estos sujetos comienzan a utilizarlos con una función de requerimiento en contextos semejantes al dar-y-tomar, mientras que la función informativa asociada a los pronombres se cumple mediante nominales como el propio nombre del niño o términos como nene. De hecho, en este tipo de contextos, el adulto, una vez que los niños dominan sin problemas las reglas de los juegos que implican una acción conjunta (dar-y-tomar, meter-y-sacar, tirar-y-construir, etc.), introduce variaciones en el marcaje de sus turnos que implican el uso de pronombres personales (ahora tú, me toca a mí, ahora yo, tú, etc.). Además, en general, incluye este tipo de formas en situaciones de "disputa" de los objetos dentro de la propia acción conjunta. Es decir, el adulto crea, en el propio seno de un juego determinado, subsituaciones que comportan "pelearse" por la posesión de un objeto determinado, delimitando aún más los procedimientos para requerir.

Igualmente, en estos formatos se trabajan explícitamente los pronombres demostrativos, especialmente en lo que se refiere a su referencia deíctica. Así, aparecen producciones del tipo aquel, dame éste, dame aquel, este otro, etc., que normalmente se acompañan de señalizaciones.

Por último, los términos de lugar también reciben un trato especial de forma que, especialmente en el juego de dar-y-tomar, los adultos emplean numerosas producciones en las que se trabaja explícitamente la distinción aquí/alli/ahí.

El siguiente ejemplo de M. ${ }^{2}$ del Mar obtenido en una situación de acción conjunta distinta al dar-y-tomar ejemplifica el dominio del lenguaje adquirido para controlar su acción con el adulto. 
OBSERVACION VIII: M. del Mar $(1 ; 5,19)$

(M.' del Mar y su madre se disponen a jugar juntas)

(M. habla con Ex.)

\begin{tabular}{ll} 
Ma: aqui/ & (está sentada en el sue- \\
$\mathrm{x} / \mathrm{M}$ & lo) \\
Ma: aqui/ & \\
\hline $\mathrm{M}$ & \\
Ma: aqui/ & $\begin{array}{l}\text { (apoya una mano en el } \\
\text { suelo) }\end{array}$ \\
\hline $\mathrm{M}$ & \begin{tabular}{l} 
s \\
\hline
\end{tabular}
\end{tabular}

M: M. ${ }^{2}$ del Mar/ $\mathrm{Ma}$

Ma: aquíl (se levanta)
$M$

$M: M .^{2}$ del Mar/ $M a$

Ma: aquí/
$\mathbf{x} / \mathbf{x} / \mathbf{x} / \mathrm{M}$

$\mathrm{M}$ : ahora/ aqui $/ \mathbf{x} /$

aquí (:)/ $\mathrm{Ma}$

(se sienta enfrente de Ma.)

(coloca seis cubos uno $\mathrm{M}$ : ¿qué hacemos?/ los encima de otro) cubitos primero/:hacemos los cubitos?/ torre

(rie, M )

Ma: $\operatorname{ah}(:) / M$

(rie, $\mathrm{M}$ )

Ma: ya está/

(ríe)

(aplaude)

M: ya está/ Ma

M: ay/¿qué vas a hacer?/

$\mathrm{Ma}$

(se incorpora un poco,

M: huy (:)/ Ma

(pega un manotazo a la torre, ríe)

(levanta los brazos) $\mathrm{M}$ : huy (:)/ $\mathrm{Ma}$

(recoge los cubos)

M: ahora otra vez/Ma

(quieta, $\mathrm{M}$ )

(reconstruye la torre)

(coloca el último cubo)

M: ya está// $\mathrm{Ma}$

torre )

(levanta los brazos)

M: ya está/uh (:)/ $\mathrm{Ma}$

(busca los cubos)

(comienza a construir la $\mathrm{M}$ : otra vez/venga/ torre)

Otra vez/ $\mathrm{Ma}$

(coloca un cubo)

M: uno/ torre

(coloca un cubo)

$\mathrm{M}: \operatorname{dos} /$ torre

(coloca un cubo)

$\mathrm{M}:$ tres/ torre

(coge un cubo)

M: y ahora este/

Ma: ya está/ $\square$

(tira la torre, rie)

$$
\begin{aligned}
& \text { (coge un cubo, } \mathrm{M} \text { ) } \\
& \text { (quieta, } \mathrm{M} \text { ) } \\
& \text { (coloca el cubo que } \\
& \text { tiene, torre) }
\end{aligned}
$$


Ma:ya está $\sim(\sim$ entre la torre y el entorno, se adelanta hacia la torre)

(coloca los cubos que fal- $\mathrm{M}: \mathrm{no} /$ todavía no/ y este tan) aqui// torre

(levanta los brazos)

$$
\begin{aligned}
& \text { M: ya está/ Ma } \\
& \text { M: ya está/ } \mathrm{Ma} \\
& \text { M: oh }(:) / \mathrm{Ma}
\end{aligned}
$$$$
\text { Ma: ya estál (aplaude) }
$$$$
\mathrm{M}
$$$$
\text { Ma: ya está/ (aplaude) }
$$

(da un manotazo a la torre, cierra los ojos)

(ríe, recoge los cubos)

En la secuencia interactiva se observa el dominio del lenguaje por parte de M. ${ }^{2}$ del Mar que le permite establecer el lugar donde quiere interactuar, asi como el momento en que toma un turno en la acción conjunta. Vila, Cortés y Zanón (1986) analizaron el formato de «lectura de libros» entre M. ${ }^{2}$ del Mar y su madre en relación con la adquisición de nominales y la orientación del primer lenguaje de la niña, concluyendo que la madre de M. ${ }^{2}$ del Mar tendía a emplear en la atención conjunta las pautas de la acción conjunta, lo cual comportaba una primera orientación expresiva en el habla de su hija. Además, constataban que en dicho contexto el tipo de pronombres utilizados tendían a ser neutros (esto, eso), de acuerdo con las propias características del contexto. Sin embargo, en la acción conjunta la pronominalización se realiza incorporando género y número (éste, éstos, esas, etc.) posibilitando que la niña reconozca las formas lingüísticas que marcan distintos significados. Tal y como señalan Bruner (1983b) y Slobin (1986), en la acción conjunta se comparte un mismo campo semántico entre el adulto y el niño, permitiendo al niño mediante algo parecido a un mecanismo de alerta reconocer las marcas lingüísticas que denotan los significados compartidos.

La siguiente observación muestra la diversidad de formas lingüísticas que pueden aparecer en la acción conjunta a lo largo de una corta secuencia interactiva.

OBSERVACION IX: M.² del Mar $(1 ; 5,19)$

(M. ${ }^{2}$ del Mar y su madre juegan a meter-y-sacar con un conjunto de anillas)

$$
\mathrm{M}: \mathrm{M} .^{2} \text { del Mar/ } \mathrm{Ma}
$$

(extiende la mano abierta $\mathrm{M}$ : dame una/que la mehacia Ma.)
M: dame una/que la me
ta/a a ver/una/ Ma

M: ¿cuál me das?/

$\mathrm{Ma}$ (coge dos anillas, una en cada mano)

(alarga las dos, $\overline{\mathrm{M}}$ ) 
anilla en una mano de $\mathrm{Ma}$

M: es la más grande de todas/ésta/

$\mathrm{x} / \mathrm{x} / \mathrm{x} / \mathrm{x} /$ anilla

\section{(muestra las anillas, $\mathrm{M}$ )}

(tira las anillas al suelo)

(encoge los brazos, M )

(gira la cabeza, anilla ) me/anilla

dame aquella rodona ("redonda")/dame/ dámela/ anilla

(se arrastra, coge la anilla) M: ésta/ anilla

(introduce la anilla en un palo)

Esta observación muestra el marcaje lingüístico del adulto que capacita al niño para establecer distinciones como la singularidad versus la pluralidad o para dominar la referencia cambiante en los términos de lugar.

El contexto de dar-y-tomar, dadas sus características, aparece como una situación potente para poder determinar la primera orientación infantil. Mila (1984), tras estudiar las primeras 50 palabras de M.' del Mar, Roger y Emilio, comprobó que M. ${ }^{2}$ del Mar tenía una orientación expresiva, mientras que Emilio era claramente referencial, situándose Roger en una posición intermedia. Si los factores interactivos, tal y como proponemos, subyacen a la aparición del primer lenguaje, es probable que se encuentren diversas formas de referencia en la acción conjunta por parte de los adultos, de forma que la madre de $\mathrm{M}^{2}$ del Mar debe tender a utilizar un número mayor de pronombres que las madres de Roger y, especialmente, de Emilio.

La Tabla IV presenta la distribución de los ofrecimientos, incitaciones a la acción y requerimientos de nuestras tres madres según empleen un nominal o un pronominal. Ciertamente, los datos no son comparables entre

TABLA IV

Distribución de las referencias nominales y pronominales en los ofrecimientos, incitaciones a la acción y requerimientos de las madres de M.` del Mar, Roger y Emilio en el juego de dar-y-tomar

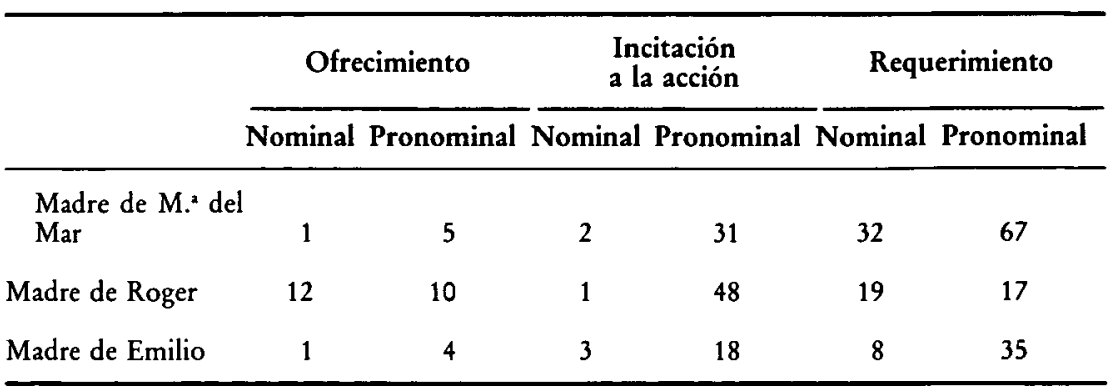


sí directamente, ya que Roger fue observado a lo largo de 17 meses. mientras que M.' del Mar sólo lo fué a lo largo de 10 meses. Sin embargo, si tenemos en cuenta que las referencias pronominalizadas se introducen relativamente tarde en el contexto los datos pueden sernos de utilidad. Finalmente, debemos señalar que hemos suprimido de los ofrecimientos la forma toma o ten que, como hemos visto, eran las más importantes y la forma dame de los requerimientos.

La comparación estricta de resultados, como ya hemos señalado, no es posible, aunque éstos presentan una tendencia en favor de la madre de $\mathrm{M}^{2}$ del Mar a realizar un número mayor de referencias pronominales. Si comparamos el habla de nuestras tres madres a partir de los 14 meses de sus hijos, encontramos que la madre de M. ${ }^{2}$ del Mar emplea significativamente más pronombres que las madres de Roger y Emilio. Por el contrario, la madre de Emilio emplea significativamente más nominales que las madres de M. ${ }^{2}$ del Mar y Roger, no diferenciándose éstas en este último aspecto.

Los resultados evidencian la importancia de la interacción en la orientación del primer lenguaje infantil, de forma que tanto $M .^{2}$ del Mar como Emilio correlacionan en su expresividad/referencialidad con el tipo de referencias que los adultos de su entorno realizan en relación a la acción conjunta.

Finalmente, destacamos otro aspecto de los resultados: el uso de la referencia deíctica. En las situaciones de dar-y-tomar las tres madres de nuestros sujetos emplean los términos ten/dame para marcar los diferentes segmentos de la acción conjunta. Ciertamente, como hemos visto, la madre de Roger prefiere utilizar el término toma, que también es utilizado por las madres de M.' del Mar y de Emilio. Sim embargo, estas últimas diferencian claramente su uso. Así, ambas emplean toma en la fase de ofrecimiento o de mantenimiento de la atención, mientras que emplean ten cuando traspasan un objeto desde sus manos a las de sus hijos. No es extraño, por tanto, que dado que el esfuerzo inicial de nuestras madres es convertir a sus hijos en unos expertos receptores, éstos escuchen repetidamente el término ten marcando el segmento de acción en el cual reciben un objeto y, consecuentemente, comiencen a usarlo para marcar dicho aspecto de la acción. Por eso, se equivocan en su uso y emplean ten tanto para traspasar como para requerir. La Tabla V muestra los errores de M.' del Mar y Emilio en su uso.

Por una parte, aparece un uso correcto correspondiente al momento en que nuestros sujetos traspasan un objeto desde sus manos a las manos del adulto que se corresponde con el uso que de dicho término hacen los adultos de su entorno. Por la otra, también emplean ten para requerir, ya que su resultado, en último término, se corresponde con un segmento de acción en el que un objeto pasa de una mano a otra. Es decir, ni M. ${ }^{2}$ del Mar ni Emilio emplean la referencia cambiante que presupone comprender que el marcaje de un mismo segmento de acción depende de la perspectiva del que lo realiza. Así, el receptor debe emplear dame, mientras que el agente utiliza ten.

M. ${ }^{2}$ del Mar emplea incorrectamente también el término tú aplicándoselo como referencia a sí misma en fórmulas como ahora tú para reivindicar su derecho a realizar una acción o emplea el pronombre en forma aislada acompañándolo de señalizaciones hacia sí misma para requerir, por ejem- 
TABLA V

Porcentaje de usos del término ten como requerimiento por parte de M. del Mar y Emilio en el contexto de dar-y-tomar

\begin{tabular}{lccc}
\hline & M.' del Mar & \multicolumn{2}{c}{ Emilio } \\
\cline { 1 - 2 } Edad & $\begin{array}{c}\text { Porcentaje de } \\
\text { usos incorrectos }\end{array}$ & Edad & $\begin{array}{c}\text { Porcentaje de } \\
\text { usos incorrectos }\end{array}$ \\
\hline $1 ; 2,2$ & 0 & $0 ; 11,15$ & 0 \\
$1 ; 2,14$ & 0 & $1 ; 0,10$ & 0 \\
$1 ; 3,9$ & 0 & $1 ; 1,1$ & 0 \\
$1 ; 4,5$ & 0 & $1 ; 4,9$ & 0 \\
$1 ; 5,19$ & 8,33 & $1 ; 4,24$ & 50,00 \\
$1 ; 7,1$ & 0 & $1 ; 5,20$ & 42,31 \\
$1 ; 7,25$ & 0 & $1 ; 6,19$ & 10,53 \\
$1 ; 8,8$ & 0 & $1 ; 8,14$ & 0 \\
$1 ; 8,29$ & 0 & $1 ; 8,27$ & 0 \\
$1 ; 9,18$ & 0 & $1 ; 10,11$ & 20,00 \\
$1 ; 10,23$ & 0 & $1 ; 10,20$ & 15,00 \\
$1 ; 11,17$ & 0 & $1 ; 11,12$ & 0 \\
$2 ; 0,3$ & 0 & $2 ; 0,2$ & 0 \\
\hline
\end{tabular}

plo, que le dejen bajar sola por la escalera sin que obligatoriamente tenga que ir de la mano de un adulto.

Sin embargo, el uso de ten como requerimiento es claramente diferente en M. ${ }^{2}$ del Mar y Emilio (véase Tabla V). Probablemente, la razón de dicha discrepancia se encuentre en el trato que los adultos de su entorno dan a dichos "errores». La siguiente observación es relevante.

OBSERVACION X: Emilio $(1 ; 5,20)$

(Emilio y su madre están sentados delante de una mesa)

E: ten/
lápiz cextiende el brazo ha-
cia un lápiz)

M: ten que/ E

$$
\begin{aligned}
& \text { lápiz } \\
& \text { E: ten } / M
\end{aligned}
$$

M: no/ se dice dame/ $\mathrm{da} / \mathrm{me}$ E

$$
\mathrm{E}: \operatorname{ten} / \mathrm{M}
$$

$\mathrm{M}: \mathrm{no} / \mathrm{da} / \mathrm{me} / \mathrm{E}$

$$
\mathrm{E}: \operatorname{ten} / \mathrm{M}
$$

M: se dice dame/ E

$$
\begin{aligned}
& \text { lápiz } \\
& \mathrm{E}: \text { ten/x/ } \\
& \mathrm{M}
\end{aligned}
$$

$\mathrm{M}: \mathrm{no} / \mathrm{E}$

$\mathrm{M}:$ ¿qué?/E $\mathrm{E} \quad \mathrm{E}: \operatorname{ten} / \mathrm{M}$




\section{$\mathrm{E}: \operatorname{ten}(:) /$}

$\mathrm{M}$

M: es que no sé qué quieres/ E

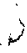

$$
\mathrm{E}: \operatorname{ten}(:) / \mathrm{M}
$$

(coge el lápiz)

da el lápiz a E.)
M: se dice dame/ esto/ $\mathrm{E}$

M: se dice dame/ E (coge el lápiz)

(manipula el lápiz)

E: ten $/ \bar{M} \quad$ (traspasa el lápiz a M.)

La madre de Emilio tiende a no aceptar el «error» de Emilio y no se adecua a su requerimiento, buscando que éste emita el término correcto. A la vez, acompaña sus rechazos con el término no que acompaña a se dice dame, lo cual probablemente es interpretado por su hijo como una negativa a satisfacer su requerimiento, lo cual incrementa el uso del procedimiento con que inició la secuencia, es decir, ten. Por tanto, los esfuerzos maternos más que posibilitar el cambio de perspectiva de Emilio aumentan la probabilidad de que emplee ten como requerimiento. Por su parte, la madre de M. ${ }^{2}$ del Mar siempre se acomoda a los requerimientos de su hija y a lo sumo emplea fórmulas del tipo ¿qué quieres que te dé?, siendo más efectiva al situar el uso de ten/dame en el dominio por parte de su hija del cambio de perspectiva en la acción conjunta más que en un proceso exclusivamente imitativo.

\section{CONCLUSIONES}

El análisis de la acción conjunta especificado en el estudio del juego de dar-y-tomar muestra su importancia para la elaboración por parte del niño de un buen número de nociones que tienen una correspondencia lingüística, permitiendo su dominio y, a la vez, la realización de una de las funciones más importantes del lenguaje: regular la conducta de otra persona.

La participación en el juego de dar-y-tomar comporta la adecuación a una serie de reglas manifestadas en «yo doy-tú recibes», luego «tú das-yo recibo". Además, debe especificarse el lugar en el cual se desea realizar los intercambios, la negación a traspasar un objeto determinado o el deseo de retener el objeto. La práctica de esta actividad permite al niño dominar el curso de la acción de forma que, a partir de un determinado momento, participa activamente en estas rutinas empleando una amplia gama de procedimientos comunicativos que, siendo gestuales al inicio, progresivamente se van lexicalizando a medida que el niño es capaz de reconocer determinados segmentos del habla del adulto como relevantes y más eficaces para cumplir las funciones que realiza mediante procedimientos más arcaicos. Aparecen producciones como ten, ya está, aquí, mío, etc., que con el tiem- 
po se generalizan a situaciones semejantes permitiendo al niño el control de su conducta en relación a la conducta del adulto.

A su, vez, dichos procedimientos lexicales son especificaciones que la gramática àporta al código lingüístico (por ejemplo, flexionado/no flexionado) para marcar diversos significados. En consecuencia, la acción conjunta, en la medida que posibilita el dominio de nociones como agente, objeto, lugar, posesión, etc., también permite acceder a las especificaciones gramaticales que las marcan.

Pero la adquisición del lenguaje no debe encararse exclusivamente como un proceso en el que se relaciona directamente una forma con un significado. Por el contrario, los problemas que tienen nuestros sujetos para usar correctamente los pares deícticos yo/tú o ten/dame muestra que una misma forma lingüística puede relacionarse con diversos significados, o viceversa, requiriendo su aprendizaje el dominio de los diferentes usos de los términos lingüísticos en el discurso.

Por último, cabe reseñar el carácter de crisol lingüístico de este tipo de contextos que aparecen como las situaciones naturales que permiten acceder al lenguaje. Una vez que el niño domina sus reglas es capaz de extenderlas a otras situaciones, generalizando sus interacciones y, por tanto, sus "comentarios" a "temas" compartidos con el adulto.

\section{Referencias}

BICKERTON, D. (1981). Roots of language. Ann Arbor, MI: Karoma.

BRUNER, J. S. (1975). The ontogenesis of speech acts. Journal of Child Language, 2, 1-19.

BRUNER, J. S. (1977). Early social interaction and language acquisition. En H. R. Schaffer (comp.): Sudies in mother-infant interaction. Londres: Academic Press.

BRUNER, J. S. (1982). The formats of language acquisition. American Journal of Semiotics, 1 (3), 1-16.

BRUner, J. S. (1983a). Child's talk. Learning to use language. Oxford: Oxford University Press.

BRUNER, J. S. (1983b). The acquisition of pragmatic commitments. En R. M. Golinkoff (comp.): The transition from prelinguistic to linguistic communication. Hillsdale, N. J.: Lawrence Erlbaum Associates.

GónZÁlEZ, A. M. y VILA, I. (1986). Estudio descriptivo de la adquisición de la referencia personal en castellano desde un año a tres años de edad. Documento inédito. Departamento de Psicologia General. Universidad de Barcelona.

DE LEMOS, C. (1981). Interactional processes in the child's construction of language. En W. Deutsch (comp.): The chil's construction of language. Londres: Academic Press.

MuNOZ, C. y ViLA, I. (1985). Es mío no: no es tuyo. Emergence and use of first and second reference in Spanish. Some observational data. En P. Fletcher y M. Garman (comp.): Child Language Seminar. Papers 1985. Reading: University of Reading.

MuÑoz, C. y ViLA, I. (1986). Adquisición de los pronombres de primera y segunda persona en castellano. En F. Fernández (comp.): Pasado, presente y futuro de la lingüística aplicada en España. Valencia: Universidad de Valencia.

NELSON, K. (1983). The conceptual basis for language. En Th. B. Seiler y W. Wannenmacher (comps.): Concept development and the development of word meaning. Berlin: Springer.

SAVASIR, I. y GEE, J. (1982). The functional equivalents of the middle voice in child language. Proceedings of the Berkely Linguistic Society. Berkeley: Universidad de California.

Siguan, M.; Colomina, R. y VILA, I. (1986). Metodologia per a l'estudi del llenguatge infantil. Vic: EUMO.

SLOBIN, D. I. (1986). Crosslinguistic evidence for the Language-Making Capacity. En D. I. Slobin (comp.): The crosslinguistic study of language acquisition, II. Hillsdale, N. J.: Lawrence Erlbaum Associates.

VILA, I. (1984). La competencia comunicativa en los dos primeros años de vida. Tesis Doctoral inédita. Departamento de Psicologia General. Universidad de Barcelona. 
VILA, I. (1987). Notas acerca de un modelo sobre la ontogénesis del lenguaje. En M. Siguan (comp.): Actualidad de Lev. S. Vigotski. Barcelona: Anthropos.

VILA, I.; CORTÉS, M. y ZANON, J. (1986). Baby-talk and child designation in the *book-reading " context. En R. Crawley, R. Stevenson y M. Tallerman (comp.): Proceedings of the Child Language Seminar 1986. Durham: Universidad de Durham.

\section{APENDICE $I$}

\section{Normas de transcripción}

1. La transcripción se realiza según cuatro columnas. La primera recoge las conductas no verbales del adulto, la segunda su lenguaje, la tercera el lenguaje del niño y la cuarta sus conductas no verbales.

2. Los adultos se marcan con las letras $\mathbf{M}$ (madre), $\mathbf{P}$ (padre) y Ex (experimentador). Los sujetos, con la primera letra de su nombre, excepto M.' del Mar, que recibe el símbolo Ma.

3. Todas las conductas no verbales se expresan en presente. Cuando aparecen diferentes conductas no verbales simultáneas se recogen una detrás de la otra separadas por una coma.

4. Cada pausa en el lenguaje que emite el adulto o el niño se marca con el símbolo (/). La repetición de una misma emisión se marca con el símbolo (x). Finalmente, una emisión que recibe un acento prolongado en su parte final se marca con (:). Por ejemplo, uиuиub se simboliza como ub (:).

5. Cada producción lingüística se acompaña de la mirada que dirige el emisor una vez que se ha transcrito la emisión. La mirada se recoge en un cuadro, incluyendo en su interior bien el símbolo que denota la persona a la que se está mirando, bien el nombre del objeto hacia el cual el interlocutor dirige su mirada. Si la mirada alterna de un punto a otro, se especifica con el símbolo $(\sim)$, a la vez que se expresan los dos puntos entre los que alterna la mirada. El símbolo $(\cdot)$ significa que el interlocutor dirige su mirada hacia la cámara que registra las conductas.

6. Las señalizaciones se expresan mediante una flecha $(\rightarrow)$, colocándose encima de la producción lingüística que acompañan. Encima de la flecha se coloca el lugar hacia donde señala el sujeto, siguiendo los mismos símbolos que para la mirada.

7. Todas las conductas no verbales se incluyen entre paréntesis. 